\title{
Community orients program for the control of rheumatic diseases Copcord project in Brazil and Latin America
}

There is an urgent need for high quality data about the magnitude and impact of rheumatic disorders in developing countries, specially in Latin America. Such data can bring attention to the substantial health impact of this group of disorders and can inform decisions regarding appropriate services for detection and treatment.

In order to evaluate the burden of illness attributable to rheumatic disorders in Latin-America a group of rheumatologists and clinical epidemiologists (all members of the International Clinical Epidemiology Network Program) decided to use the WHO-ILAR-COPCORD approach.

After initial group discussions the original COPCORD Questionnaire was slightly modified aiming at the achievement of a greater face and content validity when applied to a Latin-american environment. A Pan American League of Associations for Rheumatology (PANLAR) english version was defined.

As every crosscultural project involves the uses of language and potential problems may arise with the translation of the most simple sentence, the investigators decided to translate the instrument into Portuguese and Spanish as well as to evaluate the new instrument measurement properties.

The following study phases were conducted in Brazil, Chile and Mexico:

1. Translation of the instrument

- Primary translation;

- Evaluation of the primary translation;

- Evaluation of the cultural equivalence;

- Definition of the Portuguese/Spanish version.

2. Evaluation of Reliability - Hospital based sample

- Intra-observer;

- Test-retest.

3. Community validation study

- Definition of sensitivity;
- Definition of specificity;

- Definition of the number of phisical exams avoided.

The objective of this validation study was ultimately to evaluate the sensitivity and specifity of the Portuguese and Spanish versions of the Ilar/Copcord Core Questionnaire in terms of screenig rheumatic diseases.

In Brasil, six streets of one district of Embu were randomly selected and had 76 houses chosen also at randon and visited by interviewees. 204 individuals were interviewed and examined $(110$ females, age $16-81$, mean $=38$ years and 94 males, age 16-79, mean $=41$ years). One hundred and seven individuals reported pain tenderness, swelling or stifness in their bones, muscles or joints (50 at present and 57 in the past). Of these, after the physical examination, 68 individuals had 72 diseases diagnosed.

In adittion, 18 individuals (8.8\%) reported some disability due to the rheumatic disease at the time of the interview. The Health Assessment Questionnaire Scores ranged from 0.13 to 1.50 , with a mean value of 0.62 .

Based on these data, the Portuguese version of the Ilar/ Copcord Core Questionnaire presented a sensitivity of $92 \%$ and a specifity of $70 \%$. The prevalence of rheumatic disease in population screened and examined was $33 \%$. By using this screening questionaire 499 physical exams per 1000 individuals interviewed can be avoided. Similar studies with coinciding results were reported in Chile and Mexico.

In summary, in Latin America, a rigorous process of cross-cultural adaptation has been applied to the ILAR/ COPCORD Questionnaire and it has been validated against a full clinical exam by a rheumatologist. Also, the questionnaire is easy to administer, acceptable and relatively inexpensive, and the sequential approach of COPCORD program offers increased efficienly in large scale survey research that aim to estimate the prevalence of specific rheumatic diagnoses. 